\title{
(6) OPEN ACCESS \\ Estimating prevalence trends in adult gonorrhoea and syphilis in low- and middle-income countries with the Spectrum-STI model: results for Zimbabwe and Morocco from 1995 to 2016
}

\author{
Eline L Korenromp, ${ }^{1}$ Guy Mahiané, ${ }^{2}$ Jane Rowley, ${ }^{3}$ Nico Nagelkerke, ${ }^{4}$ \\ Laith Abu-Raddad, ${ }^{5}$ Francis Ndowa, ${ }^{6}$ Amina El-Kettani, ${ }^{7}$ Houssine El-Rhilani, ${ }^{8}$ \\ Philippe Mayaud, ${ }^{9}$ R Matthew Chico, ${ }^{9}$ Carel Pretorius, ${ }^{2}$ Kendall Hecht, ${ }^{2}$ Teodora $\mathrm{Wi}^{10}$
}

- Additional material is published online only. To view please visit the journal online (http://dx.doi.org/10.1136/ sextrans-2016-052953).

${ }^{1}$ Avenir Health, Geneva,

Switzerland

${ }^{2}$ Avenir Health, Glastonbury,

Connecticut, USA

${ }^{3}$ London, UK

${ }^{4}$ Malawi-Liverpool Wellcome

Trust, Blantyre, Malawi

Weill Cornell Medical

College-Qatar, Cornell

University, Doha, Qatar

${ }^{6}$ Skin \& Genito-Urinary Medicine Clinic, Harare, Zimbabwe

${ }^{7}$ Ministry of Health, Direction de l'Epidémiologie \& Service de Maladies Sexuellement Transmissibles, Rabat, Morocco ${ }^{8}$ UNAIDS Morocco Country Office, Rabat, Morocco ${ }^{9}$ London School of Hygiene and Tropical Medicine, London, UK ${ }^{10}$ Department of Reproductive Health and Research, World Health Organization, Geneva, Switzerland

\section{Correspondence to}

Dr Eline L Korenromp

Avenir Health, 1 route de Morillons/150 Route de Ferney (WCC, office 164), PO Box 2100, Geneva 2 CH-1211,

Switzerland;

ekorenromp@avenirhealth.org

Received 27 October 2016 Revised 6 January 2017 Accepted 14 January 2017 Published Online First 21 March 2017

\section{CrossMark}

To cite: Korenromp EL Mahiané G, Rowley J, et al. Sex Transm Infect 2017:93:599-606.

\begin{abstract}
Objective To develop a tool for estimating national trends in adult prevalence of sexually transmitted infections by low- and middle-income countries, using standardised, routinely collected programme indicator data.
\end{abstract}

Methods The Spectrum-STI model fits time trends in the prevalence of active syphilis through logistic regression on prevalence data from antenatal clinicbased surveys, routine antenatal screening and general population surveys where available, weighting data by their national coverage and representativeness.

Gonorrhoea prevalence was fitted as a moving average on population surveys (from the country, neighbouring countries and historic regional estimates), with trends informed additionally by urethral discharge case reports, where these were considered to have reasonably stable completeness. Prevalence data were adjusted for diagnostic test performance, high-risk populations not sampled, urban/rural and male/female prevalence ratios, using WHO's assumptions from latest global and regional-level estimations. Uncertainty intervals were obtained by bootstrap resampling.

Results Estimated syphilis prevalence (in men and women) declined from $1.9 \%(95 \% \mathrm{Cl} 1.1 \%$ to $3.4 \%)$ in 2000 to $1.5 \%(1.3 \%$ to $1.8 \%)$ in 2016 in Zimbabwe, and from $1.5 \%(0.76 \%$ to $1.9 \%)$ to $0.55 \%(0.30 \%$ to $0.93 \%)$ in Morocco. At these time points, gonorrhoea estimates for women aged $15-49$ years were $2.5 \%$ (95\% Cl $1.1 \%$ to $4.6 \%$ ) and $3.8 \%(1.8 \%$ to $6.7 \%)$ in Zimbabwe; and $0.6 \%$ (0.3\% to $1.1 \%$ ) and $0.36 \%$ $(0.1 \%$ to $1.0 \%)$ in Morocco, with male gonorrhoea prevalences $14 \%$ lower than female prevalence.

Conclusions This epidemiological framework facilitates data review, validation and strategic analysis, prioritisation of data collection needs and surveillance strengthening by national experts. We estimated ongoing syphilis declines in both Zimbabwe and Morocco. For gonorrhoea, time trends were less certain, lacking recent population-based surveys.

\section{INTRODUCTION}

The global burden of sexually transmitted infections (STIs) continues to be high, with over 357 million new curable infections estimated in 2012, ${ }^{1}$ which result in high morbidity and mortality, infertility, pregnancy and congenital complications, psychosocial impacts and economic productivity losses. $^{2}{ }^{3}$ At the national level, estimates of population-based infection rates and trends in STIs are important to guide specific control strategies, programmes, advocacy and resource mobilisation. Many countries have yet to implement STI surveillance systems, and where these do exist the indicator data produced vary. These challenges, and the changing epidemiology of STIs, require new strategies and technologies to expand and improve disease surveillance at both country and global levels.

Since 1995 the WHO has generated global and regional STI prevalence estimates of four curable STIs approximately every 5 years, using progressively refined epidemiological and statistical methods. ${ }^{145}$ The most recent estimates, for 2012, were based on a Bayesian meta-analytic analysis of survey prevalence data of gonorrhoea, chlamydia and trichomoniasis among low-risk populations, and on national surveillance of syphilis seroprevalence among antenatal care (ANC) attendees. Prevalence data were adjusted for laboratory test types, and geography and age of study populations. The precision and utility of these estimates are limited by varying and uncertain data availability and quality, as well as the representativeness of survey samples. WHO also generated global and regional incidence estimates from the prevalence estimates, by adjusting for the average duration of infection, which depends upon a number of additional factors which are very uncertain, such as treatment-seeking behaviours, access to health services and state of the epidemics.

WHO estimates did not include country-specific estimates and, owing to changes in methods over time, are not appropriate for generating time trends (whether global or regional). To deal with these challenges, we have developed a country-level estimation model, which is integrated into the Spectrum suite of epidemiological estimation and health policy planning tools (http://www.avenirhealth.org/softwarespectrum.php). The Spectrum suite of programmes has been developed to support estimation of national burdens, trends, service needs and programme impact for family planning, HIV/AIDS ${ }^{6-11}$ and, more recently, tuberculosis ${ }^{12}$ and malaria. ${ }^{13} 14$

The Spectrum-STI model currently focuses on two STIs-active syphilis and gonorrhoea-in adults. Active syphilis and gonorrhoea were chosen 
as they are the two STIs for which the 2016-2021 global health sector strategy for STI control, adopted by the $69^{\text {th }}$ World Health Assembly, set impact goals. ${ }^{15}$ Also, these are the two STIs for which countries routinely collect and annually report indicator data, through the Global AIDS Response Progress Reporting (GARPR) system to the Joint United Nations Programme on HIV and AIDS (UNAIDS) and WHO. ${ }^{16-19}$

To develop the Spectrum estimation methodology, we initially collated data from 10 countries to see what type of data were readily available. ${ }^{20}$ Two of these countries were then chosen to pilot the methodology in more detail-Zimbabwe and Morocco -from which national STI experts joined the Spectrum-STI development team.

This paper provides an overview of the Spectrum-STI module, its data inputs and estimation method and assumptions, and presents results for the two pilot countries. Estimate and limitations related to input data and methodology are discussed, and we make recommendations for strengthening STI surveillance to facilitate and improve future estimations and evaluation of trends.

\section{METHODS}

\section{Overview}

National prevalence estimates were generated from available country data for syphilis, and for gonorrhoea supplemented by data from neighbouring countries, using standard STI indicators that most low- and middle-income countries collect and report routinely: syphilis prevalence in pregnant women attending ANC from sentinel surveys/surveillance and routine programmatic screening, reported annually through the GARPR system since 2009, and case report data on gonorrhoea and urethral discharge (UD), reported through GARPR since 20132014. ${ }^{16-18}$

Estimation used data from 1990-2015 as available, and resulting estimations covered the period 1995-2016. The method first estimates adult female prevalence, and then the corresponding adult male prevalence.

The Spectrum-STI software is available at: http:// spectrumbeta.futuresinstitute.org/. For each country there is a default database built-in, based on Avenir Health's compilation of data reported through GARPR and other prevalence survey meta-analyses for both gonorrhoea and syphilis, as described below, which the user can adapt, and use as a starting point to create and update national estimations.

\section{Syphilis prevalence}

National syphilis estimates were generated for 'probable active' infection, defined as being concurrently rapid plasma reagin (RPR)-positive and Treponema pallidum haemagglutination assay (TPHA)-positive. This is the same definition used by the $\mathrm{WHO}^{1}$ and the Institute of Health Metrics and Evaluation ${ }^{21} 22$ in their regional and global syphilis estimates.

A standardised database of the prevalence of syphilis in each country was compiled with information from:

- Country reports of routine, continuous ANC screening results, from health ministries into GARPR, ${ }^{19}$ for the indicators:

- Coverage of routine syphilis screening among women presenting for first ANC visit (or, if first visit not available, any ANC visit), among pregnant women attending for first ANC visit, global data available at: http://apps.who. int/gho/data/node.main.A1358STI?lang=en;
- The number of women screened for syphilis at first ANC visit (the denominator of the preceding indicator), global data available at: http://apps.who.int/gho/data/node.main. A1358STI?lang $=$ en;

- Prevalence of syphilis among women screened at first ANC visit (or if first visit not available, any ANC visit); global data available at: http://apps.who.int/gho/data/node. main.A1359STI?lang=en.

- Nationally representative syphilis prevalence surveys - that is, sample-based surveillance among ANC women (first-visit, if available; otherwise any visit), typically done once every 2 years over a short (eg, 2 months) time period.

- All nationally or subnationally representative general population prevalence surveys in non-ANC male and/or female general populations, which met the criteria for representativeness and quality used in the WHO 2012 regional estimations. ${ }^{1}$

All prevalence data points were adjusted for diagnostic test performance: prevalence data based on combined RPR and TPHA positivity were taken as such; those based on RPR only were adjusted downward to 0.7 -fold of the observed prevalence for ANC and family planning populations; or to 0.6 -fold for other general populations; those based on TPHA only were adjusted downward to 0.8 -fold, those based on rapid TPHA-based test in ANC populations to 0.7-fold; and data with an unknown test to 0.75 -fold the observed prevalence. This adjustment was similar to that used in WHO global and regional estimates. ${ }^{1} 2324$ The test adjustment factors were chosen in consultation with three international STI experts (Professor Charlotte Gaydos, Professor Cesar Carcamo and Professor Patricia Garcia, May 2016).

After the diagnostic test adjustment, the syphilis data were adjusted upward by $10 \%$ to account for undersampling of high-risk populations in general population surveys, as was done by WHO in their regional and global estimates. ${ }^{1}$ In addition, for those data points with $0 \%$ syphilis prevalence this value was replaced by a prevalence calculated as 1 case divided by 100 times the sample size, to allow for logistic regression and generation of uncertainty intervals (see online supplementary file 1). The sequence of these adjustments, as applied for Zimbabwe and Morocco, is shown in online supplementary file 2 .

Each study was also assigned a weighting, to reflect its assumed representativeness for the overall national ANC population in the country:

- Nation-wide ANC surveys were assigned a weight of $100 \%$, to reflect their cluster-sampling design, ensuring representativeness. Data points with fewer than the maximum number of sites were weighted downward proportionally by the relative number of sites, relative to the maximum number of sites ever sampled for national representativeness (see online supplementary file $2 a, b)$.

- Routine ANC screening data were weighted according to the coverage of the syphilis screening, as reported through GARPR. This was done to account for the nonrepresentativeness of 'routine' data points, especially in the past when coverage was still low.

- Non-ANC general population surveys were assigned a weight of $50 \%$ if based on more than 20 sites nationwide, or between $5 \%$ and $50 \%$ if based on fewer than 20 selected sites (eg, for Morocco in 1999 and 2011-2012, online supplementary file $2 \mathrm{~b}$ ). These are conservative weights, since the representativeness of these data is less clear than for ANC data. 
Estimating prevalence in ANC women

Once the database was constructed, logistic regressions were performed with calendar year as independent variable. All data were pooled - that is, we assumed there were no systematic prevalence differences between ANC women and women in the general adult population. ${ }^{1}$ Data were weighted as above and then scaled by dividing by sample size, so that the sample size did not influence the estimated prevalence level or trend but did influence confidence bounds.

For syphilis, we assumed that all qualifying data were representative - that is, no adjustments were made for possible oversampling of urban or rural sites and urban/rural prevalence differences.

CIs (ie, uncertainty ranges) were obtained using bootstrap resampling (see online supplementary file 1 ).

Finally, the estimated point prevalence and the upper bound of the 95\% CI were restricted for any year before 1995-2021 in any country at a maximum of $20 \%$, for both women and men, based on empirical data from across low- and middle-income countries since $1990 .^{1}{ }^{25}$

\section{Estimating prevalence in adult women and men}

The resulting estimated national prevalence trend for ANC women was interpreted to also indicate prevalence and trends for all women aged 15-49 years, as assumed in the WHO 2012 estimation. ${ }^{1}$ Furthermore, given a lack of prevalence data from men in most countries, we assumed that prevalence in men aged 15-49 years nationwide equals the prevalence among women attending ANC and adult women (as in WHO global estimations ${ }^{145}$ ). This was substantiated by an average male-to-female prevalence ratio found in patients with tuberculosis in sentinel surveillance data from Morocco between 2005 and 2012 of, on average, 1.06 for TPHA and 1.00 for RPR. ${ }^{26}$

\section{Gonorrhoea prevalence}

For gonorrhoea, the estimation method was based on the principle that all of the-generally sparse-gonorrhoea prevalence data from low-risk populations in a country, and the world region in which that country is located, are informative and should be used. However, studies that are more nationally representative, and closer in time to the year(s) of interest, should get a greater weight.

A database was compiled for each country drawing on gonorrhoea prevalence data identified through systematic reviews of studies and surveys of low-risk general female and male populations, done for the WHO global and regional estimates in 2005, 2008 and 2012, ${ }^{1}$ supplemented by studies included in a meta-analysis of gonorrhoea prevalence in ANC women in sub-Saharan Africa ${ }^{25}$ and those from a meta-analysis of general populations in the WHO Eastern Mediterranean region (Smolak A, Mohamoud YA, Abu-Raddad LJ. The epidemiology of gonorrhea in the Middle East and North Africa: systematic review and meta-analysis. 2016 (in preparation)). For each country the database included studies from the country itself, studies from bordering countries (https://en.wikipedia.org/wiki/List_of countries_and_territories_by_land_borders) and regional prevalence estimates for the subregion to which the country belongs. 125

Any additional studies that we became aware of, and that met the inclusion criteria of the systematic reviews (Smolak et al (in preparation)) ${ }^{1} 25$ were also included. For studies that did not specify the year of data collection, we assumed that the data were collected 3 years before the publication date.
Observed prevalence was adjusted for diagnostic test performance, using the same approach as the 2012 WHO global and regional estimates ${ }^{1}$ and other multisite meta-analyses: ${ }^{25} 27$

$$
\underset{\text { Prevalence }}{\text { Test } \text { Adjusted }}=\frac{(\text { Observed Prevalence }+ \text { Specificity }-1)}{\text { Sensitivity }+ \text { Specificity }-1}
$$

Online supplementary file 3 provides information on the sensitivities and specificities for each of the laboratory tests.

For the gonorrhoea estimation, male and female data were combined. Male prevalence data were converted into a 'data point' corresponding to that for women by applying a fixed 0.86 male-to-female prevalence ratio, the same ratio as used in the WHO global and regional estimations. ${ }^{1} 45$ Female 'data points' thus inferred from male prevalence data (eg, for Zimbabwe, from a rural population-based survey in 2001, online supplementary file $2^{28}$ ) were then merged to the female prevalence dataset, using the original male sample size, and the estimation method described below was applied to the combined dataset, estimating the full prevalence time series for women first.

Studies conducted in exclusively rural or urban sites were converted into a corresponding national prevalence, using WHO's approach to global and regional estimates, in which the rural-to-urban gonorrhoea prevalence ratio was assumed to be 0.9. ${ }^{1}$ Weighting between rural and urban prevalences was based on the country-year-specific proportion of urban population according to the United Nations Population Division ${ }^{29}$ in that year (in Zimbabwe, 32\% throughout 1995-2016; in Morocco increasing from $52 \%$ at 1995 to $60 \%$ at 2016).

To convert prevalence estimates for low-risk general populations to national overall prevalences, estimates were adjusted upward by $10 \%$ to reflect the contribution of higher-risk populations, as was also done by the WHO in their regional and global estimates. ${ }^{1}$ This seemed reasonable based on a recent meta-analysis of gonorrhoea prevalence in Eastern Mediterranean countries, where gonorrhoea prevalence in female sex workers (FSW) was on average 10-fold higher than that in women in the general population (Smolak et al (in preparation)), and FSW were estimated to represent around 1\% of the total female adult population. ${ }^{30-33}$

In addition, all data points with a test-adjusted gonorrhoea prevalence of 0 were replaced by a prevalence calculated as one case divided by 100 times the sample size.

Each study was assigned a weight, based on whether it was a prevalence study from the country itself (weight of 100\%) or from a neighbouring country (weight of 10\%). In addition, we included the latest regional estimate from the WHO (for the region applicable), assigned a $1 \%$ weight. ${ }^{1}$ Also, for countries in sub-Sahara Africa, regional estimates for either West/Central Africa or East/Southern Africa were included for the period 1990-1999 put at 1996, and for 2000-2011 put at 2005, ${ }^{25}$ each at a $1 \%$ weight.

\section{Estimating gonorrhoea prevalence in women}

- The statistical estimation considered all eligible prevalence studies at a given time $t$, in a 'moving average' approach (see online supplementary file 1 ).

For countries with reliable national case reporting for either laboratory-confirmed gonorrhoea or syndromically diagnosed cases of UD, in men, women, or both sexes combined, with at least five data points of which one or more was after 2009, national case reporting rates were used to inform the gonorrhoea prevalence trend fitting. Case reports, however, were not 
used to inform the prevalence level, as case reporting is mostly of unknown (even if stable) coverage and completeness. To this end, the estimated gonorrhoea prevalence level was 'anchored' to an average prevalence as initially estimated from prevalence studies (see online supplementary file 1)

Final estimates for women and men, and Cls

Gonorrhoea prevalence was assumed to be constant at the level estimated for the latest year with a (country, neighbour or regional) data point (country, neighbour or regional prevalence study, or case report-based prevalence estimate; 2012 for both Morocco and Zimbabwe) for all subsequent years. Similarly, we assumed gonorrhoea prevalence to be constant at the level estimated for the first data point (1991 for Zimbabwe, 1999 for Morocco) for all earlier years.

Prevalence estimates for adult men were generated from the point estimates for women by applying the WHO's estimated, globally averaged male-to-female prevalence ratio of $0.86 .^{1}$ Confidence bounds were generated by bootstrapping (see online supplementary file 1 ).

After the statistical estimation, we constrained the national gonorrhoea point estimate of prevalence (and the upper bound of the 95\% CI) for any year (past, current and future) in any country, to be at maximum $20 \%$ for women and $17 \%$ for men, based on empirical data from across low- and middle-income countries since $1990 .^{125}$

\section{RESULTS}

\section{Syphilis prevalence}

For Zimbabwe, the estimation was based on data from seven surveys conducted among women attending ANC between 2000 and 2012, and annual routine ANC programme data between 2008 and 2015 (figure 1 and online supplementary file 2a). Estimated syphilis prevalence (equal in women and men 1549 years) declined from $1.9 \%$ (95\% CI $1.1 \%$ to $3.4 \%$ ) to $1.5 \%$ (1.3\% to $1.8 \%$ ) between 2000 and 2016 .

For Morocco, data from ANC surveys (10 years over 1996-2012) and two small-scale surveys among women attending family planning clinics in 1999 and 2011-2012 both suggested a declining prevalence (figure 1 and online supplementary file $2 \mathrm{~b}$ ). Estimated syphilis prevalence declined from $1.5 \%(0.76-1.9 \%)$ to $0.55 \%(0.3-0.93 \%)$ between 2000 and 2016 .
For both countries, the prevalence estimates were most precise around 2005-2008, the mid-point of calendar years with data.

\section{Gonorrhoea prevalence}

For Zimbabwe, the estimation was based on prevalence surveys conducted in the country in 1999, 2000 and 2001, surveys in neighbouring countries Mozambique, Zambia, South Africa and Botswana conducted between 1991 and 2011, and three regional estimates (see online supplementary file $2 \mathrm{c}$ and figure 2A). Spectrum estimated a stable gonorrhoea prevalence in women of around $2.5 \%$ (1.1\% to $4.6 \%)$ over $1995-2000$, followed by a slight rise from 2001 , to $3.8 \%$ (1.8\% to $6.7 \%)$ over 2012-2016, close to WHO's regional estimate for Southern Africa $^{1}$ (figure 2). Corresponding estimates for men, based on an assumed male-to-female prevalence ratio (see 'Methods') were $2.2 \%(1.0 \%$ to $3.9 \%)$ at 2000 and $3.3 \%(1.5 \%$ to $5.7 \%)$ at 2016. Reports of patients with UD were available, but not judged of sufficiently stable coverage and completeness over time, to inform prevalence time trends.

For Morocco, data were used from national surveys in women attending ANC and family planning clinics in 1999 and 2011-2012 (see online supplementary file 2d and figure 2B). No qualifying studies were identified from any of the countries neighbouring Morocco. During on-site workshops with national experts and stakeholders held in May and September 2016, Ministry of Health representatives decided to consider Egypt as a proxy neighbouring country, in view of its similar sociobehavioural and STI healthcare situation. One survey from Egypt, conducted among women attending ANC and family planning clinics in 1999-2000, was included in the estimation. In addition, annual UD case reporting rates over 2000-2015, the period since introduction of the syndromic STI treatment approach in Morocco, were used to inform the gonorrhoea prevalence trend.

Spectrum estimated a stable gonorrhoea prevalence in women over $1995-1999$ to $0.6 \%(0.3 \%$ to $1.1 \%)$ in year 2000 , followed by slight decline over 1999-2011. For the period 20122016 the estimated female prevalence of $0.36 \%(0.1 \%$ to $1.0 \%)$ was somewhat below the WHO's estimate for the Eastern Mediterranean region $(0.62 \%) .{ }^{1}$ Corresponding estimates for men were $0.53 \%(0.3 \%$ to $0.96 \%)$ at 2000 and $0.32 \%(0.12 \%$ to $0.87 \%)$ at 2016 .
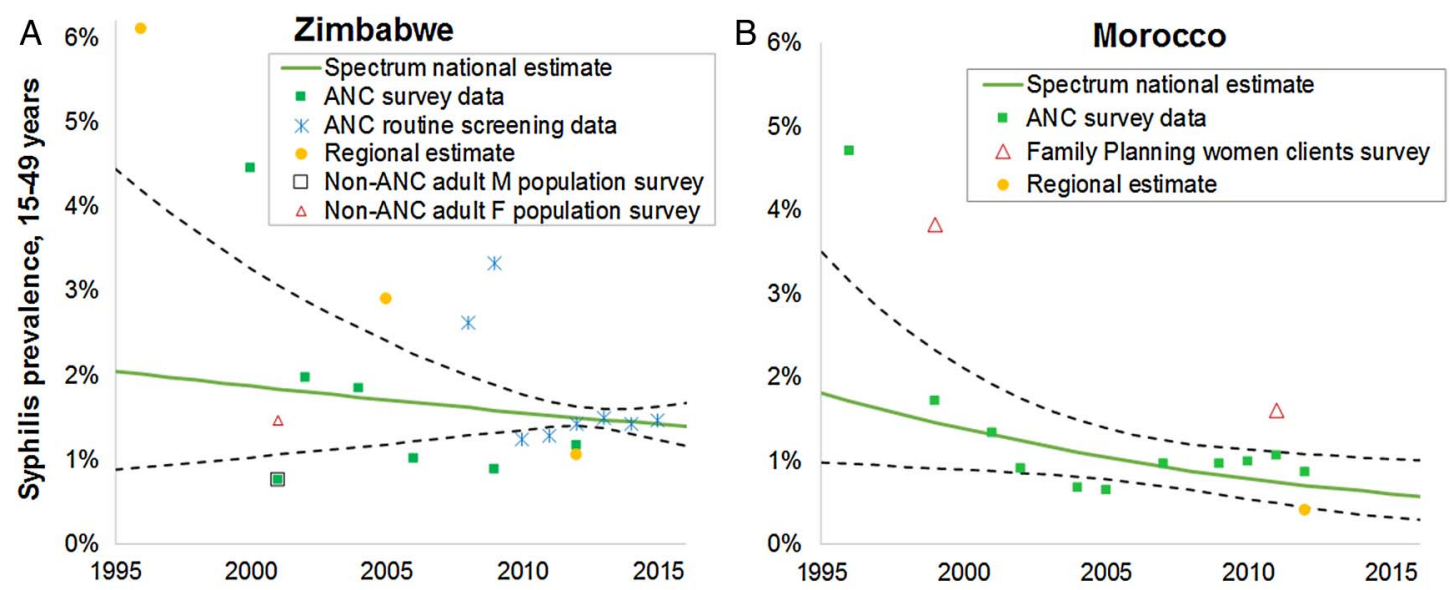

Figure 1 Spectrum-estimated national adult syphilis prevalences. Data are shown after adjustments for diagnostic test performance and missing high-risk populations, as described in the 'Methods' section. Regional estimates-shown for reference, but not used in the estimation process-are those by WHO for $2012^{1}$ for both countries, and those from a meta-analysis of antenatal care (ANC) surveys in sub-Sahara African countries for 1990-1999 (put at 1996) and 2000-2011 (put at 2005) for Zimbabwe. ${ }^{25}$ Dashed lines represent $95 \%$ Cls around the Spectrum estimate. Data shown are for women aged 15-49 years; the estimated prevalence applies to both women and men 15-49 years. 

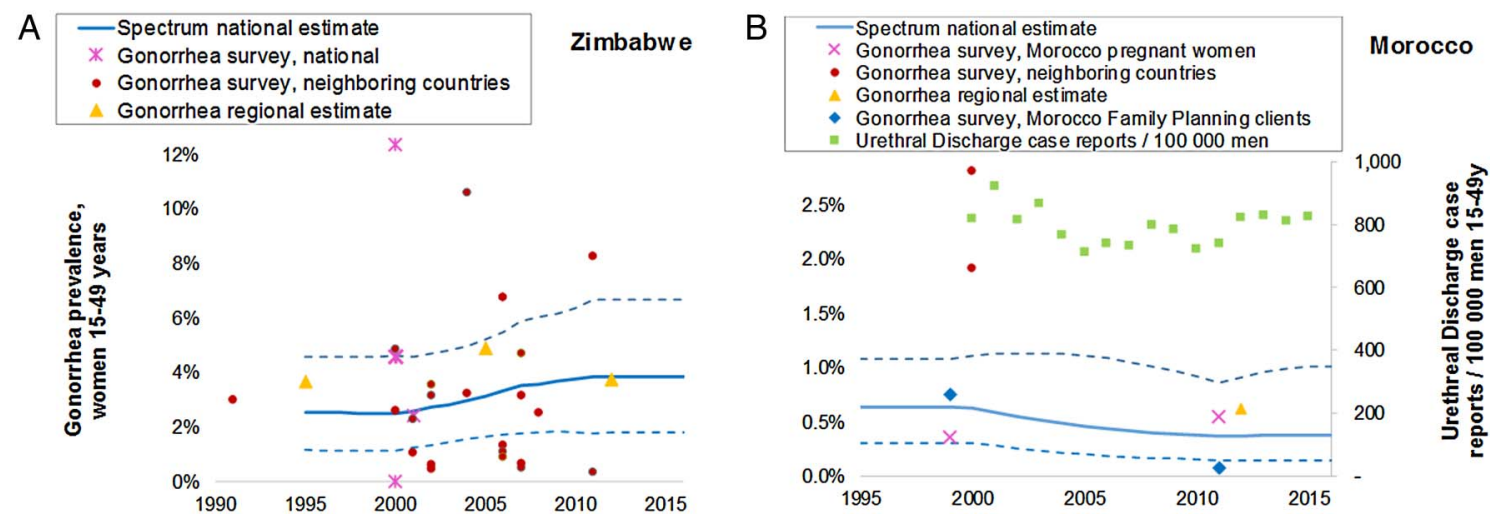

Figure 2 Spectrum-estimated national gonorrhoea prevalence in women aged 15-49 years. Data are shown after adjustments for diagnostic test performance, urban versus rural prevalence ratios and missing high-risk populations, as described in the 'Methods' section. Regional estimates (included in the estimation at a weight of $1 \%$ relative to national studies) are from the WHO for $2012,{ }^{1}$ and for Zimbabwe from a meta-analysis of antenatal care (ANC) surveys in sub-Sahara African countries for 1990-1999 (put at 1996) and 2000-2011 (put at 2005). ${ }^{25}$ Data and estimates shown are for women 15-49 years; for Zimbabwe, some additional data included in the estimation are not shown in the graph: three male survey data points from Zimbabwe; one male survey data point from South Africa; and 1 female data point from Mozambique in year 2000 at $26.8 \%$ test-adjusted prevalence; see online supplementary file $2 c$ ).

\section{Sensitivity analysis}

The principal drivers and uncertainties in estimated prevalence levels and trends were evaluated in univariate sensitivity analysis, varying in turn, diagnostic adjustment factors, weights applied to different types of data and the assumed annual dilution factor used in the moving-average trend estimation for gonorrhoea.

For syphilis, in Morocco, the diagnostic test adjustments for both RPR and TPHA, each used in about half of data points, influenced prevalence level, at 2016 in a range from $0.36 \%$ to $0.74 \%$, whereas the estimated trend of decline was robust across all diagnostic assumptions evaluated (table 1a).

For Zimbabwe, the adjustment values for rapid test and RPR, used in routine programmatic screening between 2008 and 2015 , were critical. The estimated syphilis point prevalence at 2016 decreased (from default 1.54\%) to $1.34-1.38 \%$ when assuming only $45 \%$ sensitivity for either test; whereas prevalence increased to $1.96-1.99 \%$ when assuming $100 \%$ sensitivity for either test. Furthermore, the estimated trend reversed into a time-constant value if the rapid syphilis test was assumed to be $100 \%$ sensitive, or into a slight increase over 2000-2016 (instead of the default: slight decline) if RPR was assumed 100\% sensitive. The adjustment value for TPHA did not influence Zimbabwe's syphilis level at 2016 or the (declining) time trend.

Gonorrhoea prevalence estimates were sensitive to the specificity of diagnostic techniques that employed PCR and ligase chain reaction (LCR), the most commonly used diagnostic tests: if their specificity was varied between $99.0 \%$ and $100 \%$, the estimated prevalence in Morocco at 2016 shifted in a range from $0.64 \%$ to $0.05 \%$ (table 1 b). Across all alternative assumptions tested, the estimated slight decline from 2000 to 2016 was maintained for Morocco, except when reducing the annual dilution factor to zero, which (by definition) resulted in a timeconstant prevalence estimate. For Morocco, giving more weight to regional neighbour Egypt, with two relatively high prevalence data points, increased the 2016 prevalence estimate from the best estimate of $0.36-0.43 \%$.

For Zimbabwe, similarly, the estimated gonorrhoea time trend (of increasing prevalence) was maintained across all alternative model assumptions, except when reducing the annual dilution factor to zero. The point prevalence at 2016 increased (from default $3.8 \%$, up to $4.1 \%$ ) when assuming lower specificity of
PCR and LCR tests (99.0\% instead of default 99.7\%), and prevalence decreased (to 3.6\%) when assuming 100\% specificity of PCR and LCR. Above all, Zimbabwe's gonorrhoea prevalence at 2016 was sensitive to the weight given to neighbouring country studies, increasing (to 4.5\%) when weighting neighbouring-country data each $30 \%$ (instead of default $10 \%$ ) or decreasing to $2.5 \%$ when not using neighbouring-country data.

\section{DISCUSSION}

The Spectrum-STI module is a tool that countries can use to collate STI surveillance data and interpret temporal trends in STI prevalence. Presented results for Zimbabwe and Morocco reflect collaborations with national experts and show that, even when data are limited, it is possible to make national trend estimations. In addition, the process of collating and interpreting the data systematically encourages programmes to identify key data gaps, and to prioritise actions for improving surveillance and for more strategic use of data and estimations.

In both countries, there were considerably more data for syphilis than for gonorrhoea and therefore the syphilis estimates are more robust. Results suggest an ongoing decline in the prevalence of adult syphilis in both countries, consistent with recent WHO estimates of syphilis trends in ANC women in Africa, ${ }^{24}$ and as aimed for by programmes for the elimination of congenital syphilis. ${ }^{34}$

Evidence is weaker for recent trends in gonorrhoea, which has fewer population-based data than syphilis. For Morocco, the slight decline in prevalence over 2002-2014 may be consistent with an increase in formal treatment seeking for urethral and vaginal discharge, as measured between two knowledge, attitude and practice surveys among 15-24-year-old men and women in 2007 and 2013..$^{35}$ The Spectrum-estimated decline is also consistent with a decline since 2001 in HIV incidence and increasing condom usage following expansion of Morocco's HIV response. ${ }^{33} 37$ Furthermore, gonorrhoea prevalence among women consulting clinics for vaginal discharge and/or lower abdominal pain, fell from $4.8 \%$ in $1996^{38} 39$ to $1.0 \%$ in $2007,{ }^{40}$ although the data from this selected population in selected cities were not formally used in the estimation.

The higher syphilis and gonorrhoea prevalence estimates for Zimbabwe compared with Morocco are consistent with Zimbabwe's much larger HIV epidemic. In Zimbabwe the 
Table 1 Sensitivity analysis-effect of varying (selected) assumptions and values, on national prevalence estimates for adults aged 1549 years at years 2000 and 2016: (a) syphilis; (b) gonorrhoea

\begin{tabular}{|c|c|c|c|c|c|}
\hline \multirow[b]{2}{*}{ Parameter } & \multirow{2}{*}{$\begin{array}{l}\text { Default } \\
\text { value }\end{array}$} & \multicolumn{2}{|c|}{$\begin{array}{l}\text { Alternative } \\
\text { assumption }\end{array}$} & \multirow{2}{*}{$\begin{array}{l}\text { Prevalence Zimbabwe, } \\
\text { women, at } 2000 \rightarrow 2016\end{array}$} & \multirow{2}{*}{$\begin{array}{l}\text { Prevalence Morocco, } \\
\text { women, at } 2000 \rightarrow 2016\end{array}$} \\
\hline & & Lower & Upper & & \\
\hline \multicolumn{6}{|l|}{ (a) Syphilis } \\
\hline Default best syphilis prevalence estimates & & & & $1.94 \rightarrow 1.54 \%$ & $1.48 \% \rightarrow 0.55 \%$ \\
\hline \multicolumn{6}{|c|}{ Diagnostic test performance, relative to RPR-positive plus TPHA-positive (dual positivity) as 'gold standard' } \\
\hline TPHA in ANC or FP population & 0.8 & 0.6 & 1.0 & $\begin{array}{l}\text { Lower value: } 1.73 \rightarrow 1.58 \% \\
\text { Upper value: } 2.11 \rightarrow 1.49 \%\end{array}$ & $\begin{array}{l}\text { Lower value: } 1.45 \rightarrow 0.36 \% \\
\text { Upper value: } 1.57 \rightarrow 0.74 \%\end{array}$ \\
\hline RPR & 0.6 & 0.45 & 1.0 & $\begin{array}{l}\text { Lower value: } 1.99 \rightarrow 1.38 \% \\
\text { Upper value: } 1.84 \rightarrow 1.99 \%\end{array}$ & $\begin{array}{l}\text { Lower value: } 1.22 \rightarrow 0.58 \% \\
\text { Upper value: } 2.27 \rightarrow 0.46 \%\end{array}$ \\
\hline Rapid syphilis test (TPHA-based) & 0.7 & 0.55 & 1.0 & $\begin{array}{l}\text { Lower value: } 1.90 \rightarrow 1.34 \% \\
\text { Upper value: } 1.96 \rightarrow 1.96 \%\end{array}$ & Not used \\
\hline \multicolumn{6}{|l|}{ (b) Gonorrhoea } \\
\hline Default best gonorrhoea prevalence estimates & & & & $2.5 \rightarrow 3.8 \%$ & $0.60 \rightarrow 0.36 \%$ \\
\hline \multicolumn{6}{|l|}{ Diagnostic test performance-gonorrhoea } \\
\hline Specificity PCR and LCR for gonorrhoea, women, genital fluid & $99.7 \%$ & $99.0 \%$ & $100 \%$ & $\begin{array}{l}\text { Lower value: } 2.3 \rightarrow 3.6 \% \\
\text { Upper value: } 2.7 \rightarrow 4.0 \%\end{array}$ & $\begin{array}{l}\text { Lower value: } 0.28 \rightarrow 0.11 \% \\
\text { Upper value: } 0.67 \rightarrow 0.55 \%\end{array}$ \\
\hline Specificity PCR, LCR and SDA for gonorrhoea, women, urine & $99.7 \%$ & $99.0 \%$ & $100 \%$ & $\begin{array}{l}\text { Lower value: } 2.3 \rightarrow 3.7 \% \\
\text { Upper value: } 2.7 \rightarrow 4.0 \%\end{array}$ & $\begin{array}{l}\text { Lower value: } 0.16 \rightarrow 0.09 \% \\
\text { Upper value: } 0.82 \rightarrow 0.44 \%\end{array}$ \\
\hline $\begin{array}{l}\text { Specificity PCR, LCR and SDA for gonorrhoea, women, urine and } \\
\text { genital fluid }\end{array}$ & 99.7 & $99.0 \%$ & $100 \%$ & $\begin{array}{l}\text { Lower value: } 2.1 \rightarrow 3.6 \% \\
\text { Upper value: } 2.8 \rightarrow 4.1 \%\end{array}$ & $\begin{array}{l}\text { Lower value: } 0.11 \rightarrow 0.05 \% \\
\text { Upper value: } 0.88 \rightarrow 0.64 \%\end{array}$ \\
\hline $\begin{array}{l}\text { Sensitivity of PCR/LCR and SDA for gonorrhoea, women, urine and } \\
\text { genital fluid }\end{array}$ & $\begin{array}{l}93.3 \% \\
91.6 \% \\
91.6 \%\end{array}$ & $80 \%$ & $100 \%$ & $\begin{array}{l}\text { Lower value: } 2.8 \rightarrow 4.3 \% \\
\text { Upper value: } 2.4 \rightarrow 3.6 \%\end{array}$ & $\begin{array}{l}\text { Lower value: } 0.67 \rightarrow 0.41 \% \\
\text { Upper value: } 0.54 \rightarrow 0.32 \%\end{array}$ \\
\hline Sensitivity of culture for gonorrhoea, women & $75.7 \%$ & $50 \%$ & $90 \%$ & $\begin{array}{l}\text { Lower value: } 2.7 \rightarrow 4.1 \% \\
\text { Upper value: } 2.6 \rightarrow 3.8 \%\end{array}$ & Not used \\
\hline \multicolumn{6}{|l|}{ Other parameters in the gonorrhoea prevalence estimation } \\
\hline $\begin{array}{l}\text { Weight of UD case report data points, relative to national prevalence } \\
\text { studies (the latter at } 100 \% \text { ) }\end{array}$ & $25 \%$ & $1 \%$ & $50 \%$ & Not used & $\begin{array}{l}\text { Lower value: } 0.70 \rightarrow 0.26 \% \\
\text { Upper value: } 0.54 \rightarrow 0.37 \%\end{array}$ \\
\hline $\begin{array}{l}\text { Weight of neighbouring country prevalence surveys, relative to } \\
\text { national prevalence studies }\end{array}$ & $10 \%$ & $0 \%$ & $30 \%$ & $\begin{array}{l}\text { Lower value: } 2.2 \rightarrow 2.5 \% \\
\text { Upper value: } 3.1 \rightarrow 4.5 \%\end{array}$ & $\begin{array}{l}\text { Lower value: } 0.43 \rightarrow 0.31 \% \\
\text { Upper value: } 0.82 \rightarrow 0.43 \%\end{array}$ \\
\hline Annual dilution factor in moving average estimation for gonorrhoea* & $20 \%$ & $0 \%$ & $40 \%$ & $\begin{array}{l}\text { Lower value: } 3.0 \% \text { constant } \\
\text { Upper value: } 2.4 \rightarrow 6.0 \%\end{array}$ & $\begin{array}{l}\text { Lower value: } 0.49 \% \text { constant } \\
\text { Upper value: } 0.67 \rightarrow 0.37 \%\end{array}$ \\
\hline
\end{tabular}

${ }^{*}$ The annual dilution factor weights the contribution of each data point to the estimation for other years than the study year (earlier and later, within the period from first to last data point) downward, by a fixed proportion (default value: $20 \%$ ) for each additional year away from the estimated year.

ANC, antenatal care; FP, family planning; LCR, ligase chain reaction; RPR, rapid plasma reagin; SDA, strand displacement amplification; TPHA, Treponema pallidum haemagglutination assay; UD, urethral discharge.

Spectrum model estimated a slight increase in the prevalence of gonorrhoea between 2000 and 2010, and while syphilis prevalence decreased between 1995 and 2016 this decline was less precipitous than in estimates for the Southern Africa region as a whole $^{25}$ (figure 1A) or for Morocco (figure 1B). It remains unclear whether the estimated rise in gonorrhoea prevalence in Zimbabwe is a true rise; the rise in prevalence from 1995 to 2016 estimated by Spectrum might have followed a decrease over the years just before 1995. Both gonorrhoea and syphilis might have fallen in the early 1990s (from higher levels in the 1980s), in conjunction with the reversal and fall of HIV incidence after its peak around 1990, ${ }^{41} 42$ attributed to an increase in condom usage and other behavioural risk reductions in the early 1990s. ${ }^{43-46}$

The stronger evidence for a decline in syphilis compared with that in gonorrhoea in Morocco and Zimbabwe, as well as observed syphilis declines in ANC populations in the context of programmes of congenital syphilis elimination globally, ${ }^{34}$ suggest that WHO's global STI strategy target of a 90\% reduction in infection incidence is probably more feasible for syphilis than for gonorrhoea. This reflects the different natural histories, and response to treatment and prevention interventions between these infections, with notably a higher proportion of syphilis cases being symptomatic ${ }^{1} 47^{48}$ and the emerging impact of routine ANC syphilis screening being rolled-out. ${ }^{34}$ Also the widespread use of syndromic management of genital ulcer disease with benzathine penicillin has probably contributed to declining syphilis rates, especially in high-HIV countries in Southern and Eastern Africa, including Zimbabwe, ${ }^{49}$ whereas the effectiveness and impact of gonorrhoea treatment may be diminishing in some countries owing to rising microbial resistance against some first-line treatments. ${ }^{50}$

\section{Limitations and next steps}

The biggest challenge when using a data-driven model like Spectrum is the quantity, quality and representativeness of data available for use.

For gonorrhoea, burden estimation would be facilitated by more frequent prevalence surveys-but with many competing health and surveillance priorities this is unlikely to happen. Improved, standardised gonorrhoea and UD case reporting, with ongoing monitoring of healthcare access, coverage and reporting completeness and STI aetiologies (eg, Rietmeijer ${ }^{51}$ ), will strengthen the estimation of trends. However, given timevarying, sometimes high but unknown extents of asymptomatic gonorrhoea, this approach is not a good basis for inferring prevalence levels even in countries with high STI treatment coverage and reporting completeness. Future refined methods 
might rather try to estimate burdens and trends in gonorrhoea and chlamydia in tandem, as there are more abundant chlamydia prevalence data, including from adolescent populations targeted by chlamydia screening programmes being expanded (as recommended in the WHO's global STI strategy 2016$2021^{15}$ ), while noting that these infections differ in natural history and resulting epidemic dynamics, even if they share underlying risk behaviours.

The fixed 10\% upward adjustment for high-risk populations typically missing from population prevalence surveys is crude. However, in Morocco the comparative prevalences of gonorrhoea and syphilis in FSW in 2007 and $2012^{32} 52$ combined with estimates of the number of $\mathrm{FSW}^{30} 3133$ suggest that $10 \%$ may be a reasonable adjustment value. In future Spectrum versions, we hope to incorporate syphilis prevalence data from key risk groups such as FSW and men who have sex with men, which many countries monitor periodically through integrated bio-behavioural surveillance. ${ }^{32} 5354$ This should improve the estimation, given variation among countries in risk gradients between these key groups and the general adult population, and variation in sizes of populations at risk.

Although not presented here, case incidence rates have been calculated for Morocco, Zimbabwe and other countries using the Spectrum-STI tool. Such estimates are important for planning of health services and in evaluation of STI healthcare access and reporting completeness. Case incidence is a key outcome also at global level: the WHO global STI strategy 2016-2021 set its impact targets as a 90\% incidence reduction from 2018 to 2030 for gonorrhoea and syphilis. ${ }^{15}$ In addition, there is interest in expanding the Spectrum model to estimate national burdens of congenital syphilis, ${ }^{2455}$ as recommended by the WHO, applying the same ANC prevalence data and female prevalence estimates already used in Spectrum. Also, trends in STI-related complications such as Ophthalmia neonatorum, estimated to occur in $2-48 \%$ of neonates born to mothers with gonorrhoea, if not receiving ocular prophylaxis, ${ }^{56}$ could be added to the next Spectrum version.

Age differentials within adult STI rates were ignored, but could be added if forthcoming age-disaggregated prevalence data suggested plausible age patterns.

In the longer term, estimates ideally would be made at provincial or district level. In some countries it may already be possible to generate temporal trends for syphilis in ANC women at subnational level. Many high-HIV countries use the Spectrum AIDS Incidence Model for province-level HIV/AIDS estimations; ${ }^{957}$ a similar process could be envisioned for syphilis estimation. In countries where syphilis rates vary considerably by geography, the resulting estimates may more powerfully detect any (upward or downward) time trends, compared with national-level estimation, where trends may get obscured or confounded if survey sites and the balance between low-prevalence and high-prevalence regions happened to change over time.

In conclusion, the new Spectrum-STI estimation method is a promising tool to improve national estimates of STI prevalence and trends over time. The model has been developed for easy use by national surveillance experts, which should help to strengthen STI surveillance, programme design, evaluation and advocacy activities at county, regional and global levels. Spectrum-based STI estimations may, in turn, inform surveillance and programme planning for HIV/AIDS, and be the basis for a future tool to evaluate STI programme impacts and cost-effectiveness.

Acknowledgements We thank Dr Alex Smolak (Weill Cornell Medical CollegeQatar) for sharing country data and meta-analysis results for gonorrhoea and syphilis prevalence in the Eastern Mediterranean region. We thank Professor Nicola Low
(University of Bern), Dr Jesus-Maria Garcia Calleja (WHO) and other participants at a technical consultation held in November 2015 for advice on modelling methods and assumptions. We thank Dr Melanie Taylor (WHO), Dr Robert McKinnon and John Stover (Avenir Health) for strategic advice and project oversight.

Contributors ELK and TW conceived the project; ELK, GM and NN designed the Spectrum approach; GM, NN and CP designed the statistical methodology; ELK, GM, NN, LA-R, FN and JR analysed results; FN, JR, LA-R, PM, RMC, AE-K and HE-R provided country data; LAR, PM, RMC, FN and TW provided biomedical modelling assumptions; $\mathrm{KH}, \mathrm{GM}$ and $\mathrm{CP}$ programmed the Spectrum user interface software; ELK and JR wrote the article; all authors analysed the final results, reached consensus in the interpretation and contributed to writing the final article.

Funding The project was funded by the WHO, Department of Reproductive Health and Research. LA-R acknowledges the support of Qatar National Research Fund (NPRP 9-040-3-008), which provided the main funding for generating the data provided for this study.

Competing interests None declared.

Provenance and peer review Commissioned; externally peer reviewed.

More info The views expressed in this paper are those of the authors and do not necessarily represent the position of Avenir Health, the WHO, the Joint United Nations Programme on HIV and AIDS, Morocco's Ministry of Health or any other affiliated organisation.

Data sharing statement All data used were provided within the manuscript and its three supplementary files.

Open Access This is an Open Access article distributed in accordance with the Creative Commons Attribution Non Commercial (CC BY-NC 4.0) license, which permits others to distribute, remix, adapt, build upon this work non-commercially, and license their derivative works on different terms, provided the original work is properly cited and the use is non-commercial. See: http://creativecommons.org/ licenses/by-nc/4.0/

\section{REFERENCES}

1 Newman L, Rowley J, Vander Hoorn S, et al. Global estimates of the prevalence and incidence of four curable sexually transmitted infections in 2012. PLOS ONE 2015; 10:e0143304

2 Holmes K. Sexually transmitted diseases. 4th edn. New York City: McGraw-Hill Medical, 2008.

3 Global Burden of Disease Study 2013 Collaborators. Global, regional, and national incidence, prevalence, and years lived with disability for 301 acute and chronic diseases and injuries in 188 countries, 1990-2013: a systematic analysis for the Global Burden of Disease Study 2013. Lancet 2015;386:743-800.

4 Gerbase AC, Rowley JT, Heymann DH, et al. Global prevalence and incidence estimates of selected curable STDs. Sex Transm Infect 1998;74(Suppl 1):S12-16.

5 World Health Organization. Global incidence and prevalence of selected curable sexually transmitted infections-2008. Geneva, 2012

6 Fabic MS, Choi Y, Bongaarts J, et al. Meeting demand for family planning within a generation: the post-2015 agenda. Lancet 2014;385:1928-31.

7 Halperin DT, Stover J, Reynolds HW. Benefits and costs of expanding access to family planning programs to women living with HIV. AIDS 2009;23(Suppl 1): S123-30

8 Stover J, Ross J. How increased contraceptive use has reduced maternal mortality. Matern Child Health J 2010;14:687-95.

9 Stover J, Brown T, Marston M. Updates to the Spectrum/Estimation and Projection Package (EPP) model to estimate HIV trends for adults and children. Sex Transm Infect 2012;88(Suppl 2):i11-6.

10 Stover J, Johnson P, Zaba B, et al. The Spectrum projection package: improvements in estimating mortality, ART needs, PMTCT impact and uncertainty bounds. Sex Transm Infect 2008;84(Suppl 1):i24-30.

11 Stover J, McKinnon R, Winfrey B. Spectrum: a model platform for linking maternal and child survival interventions with AIDS, family planning and demographic projections. Int J Epidemiol 2010;39:i7-10.

12 Houben RM, Lalli M, Sumner T, et al. TIME Impact-a new user-friendly tuberculosis (TB) model to inform TB policy decisions. BMC Med 2016;14:56.

13 Korenromp EL, Mahiané $G$, Hamilton $M$, et al. Malaria intervention scale-up in Africa: effectiveness predictions for health program planning tools, based on dynamic transmission modelling. Malar J 2016;15

14 Hamilton M, Mahiané G, Werst $\mathrm{E}$, et al. Spectrum-Malaria: a user-friendly projection tool for health impact assessment and strategic planning for malaria programs in sub-Saharan Africa. Malar J 2017; doi:10.1186/s12936-017-1705-3.

15 World Health Organization. Global health sector strategy on sexually transmitted infections, 2016-2021. Report. Geneva, 2016.

16 World Health Organization. Report on global sexually transmitted infection surveillance 2013. Geneva, 2014

17 World Health Organization. Baseline report on global sexually transmitted infection surveillance 2012. Geneva, 2013 
18 World Health Organization. A tool for strengthening STI surveillance at the country level. Geneva, 2015.

19 UNAIDS. Global AIDS response progress reporting 2015-guidance. Geneva, 2014

20 Korenromp EL, Mahiané G, Nagelkerke N, et al. eds. The Spectrum model estimating national syphilis and gonorrhea prevalence \& trends: results for 10 lowand middle-income countries, and implications for strengthening STI surveillance. 17th IUSTI World Congress; 9-12 May 2016; Marrakech.

21 Lozano R, Naghavi M, Foreman K, et al. Global and regional mortality from 235 causes of death for 20 age groups in 1990 and 2010: a systematic analysis for the Global Burden of Disease Study 2010. Lancet 2012;380:2095-128.

22 Kassebaum NJ, Bertozzi-Villa A, Coggeshall MS, et al. Global, regional, and national levels and causes of maternal mortality during 1990-2013: a systematic analysis for the Global Burden of Disease Study 2013. Lancet 2014;384:980-1004

23 Ham DC, Lin C, Newman L, et al. Improving global estimates of syphilis in pregnancy by diagnostic test type: a systematic review and meta-analysis. Int J Gynaecol Obstet 2015;130(Suppl 1):S10-14.

24 Newman L, Kamb M, Hawkes S, et al. Global estimates of syphilis in pregnancy and associated adverse outcomes: analysis of multinational antenatal surveillance data. PLoS Med 2013:10:e1001396.

25 Chico RM, Mayaud P, Ariti C, et al. Prevalence of malaria and sexually transmitted and reproductive tract infections in pregnancy in sub-Saharan Africa: a systematic review. JAMA 2012;307:2079-86.

26 Ministère de la Santé du Maroc. Surveillance sentinelle du VIH et de la syphilis. Rabat, 2005-2012

27 Orroth KK, Korenromp EL, White RG, et al. Comparison of STD prevalences in the Mwanza, Rakai, and Masaka trial populations: the role of selection bias and diagnostic errors. Sex Transm Infect 2003;79:98-105.

28 NIMH Collaborative HIV/STD Prevention Trial Group. Sexually transmitted disease and HIV prevalence and risk factors in concentrated and generalized HIV epidemic settings. AIDS 2007;21(Suppl 2):S81-90.

29 World Population Prospects: the 2015 revision [database on the internet]. 2015. http://esa.un.org/unpd/wpp/

30 Johnston LG, McLaughlin KR, El Rhilani $\mathrm{H}$, et al. Estimating the size of hidden populations using respondent-driven sampling data: case examples from Morocco. Epidemiology 2015;26:846-52.

31 Kouyoumjian SP, Haddad P, El Rhilani H, et al. National and Souss Massa Draa HIV modes of transmission analysis in Morocco 2013. Rabat: Kingdom of Morocco Ministry of Health Department of Epidemiology and Disease Control National STI/AIDS Programme, Weill Cornell Medical College in Qatar, Qatar Foundation, ONUSIDA, 2014

32 Ministère de la santé du Maroc. Integrated behavioral and biological surveillance survey among female sex workers in Agadir, Fes, Rabat and Tanger, Morocco, 2010-2011. Rabat, 2012.

33 Mumtaz GR, Kouyoumjian SP, Hilmi N, et al. The distribution of new HIV infections by mode of exposure in Morocco. Sex Transm Infect 2013;89(Suppl 3):iii49-56.

34 Wijesooriya NS, Rochat RW, Kamb ML, et al. Declines in maternal and congenital syphilis in 2008 and 2012: a health systems modelling study. Lancet Glob Health 2016:4:e525-33

35 Ministère de la santé du Maroc. Etude sur les comportements, attitudes et pratiques des jeunes en matière de VIH-sida. Rabat, 2007.

36 Ministère de la santé du Maroc. Etude sur les comportements, attitudes et pratiques des jeunes en matière de VIH-sida. Rabat, 2013.

37 Kouyoumjian SP, Mumtaz GR, Hilmi N, et al. The epidemiology of HIV infection in Morocco: systematic review and data synthesis. Int J STD AIDS 2013:24:507-16

38 Ryan A, Manhart L. Les maladies sexuellement transmissibles au Maroc, prévalence des infections, évaluation de risque et arbre de décision de traitement. Rapport final. AIDSCAP Université de Washington, 1997.
39 Ryan $C A$, Zidouh A, Manhart $L E$, et al. Reproductive tract infections in primary healthcare, family planning, and dermatovenereology clinics: evaluation of syndromic management in Morocco. Sex Transm Infect 1998;74(Suppl 1):S95-105.

40 Royaume du Maroc Programme National de lutte contre les IST/SIDA Direction de l'épidémiologie et des luttes contres les maladies du ministère de la santé. Etude de prévalence des IST chez les femmes qui consultent pour pertes vaginales et/ou douleurs du bas ventre. Rabat, 2008.

41 Zimbabwe Ministry of Health. Global AIDS Response Report 2016, Zimbabwe country report, reporting period January 2015-December 2015. Harare, 2016.

42 Zimbabwe National AIDS Council. Annual report 2015, Coordinating The National Response to HIV and AIDS in Zimbabwe. Harare, 2016.

43 Hallett TB, Aberle-Grasse J, Bello G, et al. Declines in HIV prevalence can be associated with changing sexual behaviour in Uganda, urban Kenya, Zimbabwe, and urban Haiti. Sex Transm Infect 2006;82(Suppl 1):i1-8

44 Gouws E, International Group on Analysis of Trends in HIV Prevalence and Behaviours in Young People in Countries most Affected by HIV. Trends in HIV prevalence and sexual behaviour among young people aged 15-24 years in countries most affected by HIV. Sex Transm Infect 2010;86(Suppl 2):ii72-83.

45 Awad SF, Abu-Raddad LJ. Could there have been substantial declines in sexual risk behavior across sub-Saharan Africa in the mid-1990s? Epidemics 2014;8: 9-17.

46 Gregson S, Gonese E, Hallett TB, et al. HIV decline in Zimbabwe due to reductions in risky sex? Evidence from a comprehensive epidemiological review. Int J Epidemiol 2010;39:1311-23.

47 Brunham RC, Plummer FA. A general model of sexually transmitted disease epidemiology and its implications for control. Med Clin North Am 1990;74:1339-52.

48 Korenromp EL, Bakker R, Gray R, et al. The effect of HIV, behavioural change, and STD syndromic management on STD epidemiology in sub-Saharan Africa: simulations of Uganda. Sex Transm Infect 2002;78(Suppl 1):i55-63.

49 Korenromp EL, White RG, Orroth KK, et al. Determinants of the impact of sexually transmitted infection treatment on prevention of HIV infection: a synthesis of evidence from the Mwanza, Rakai, and Masaka intervention trials. J Infect Dis 2005; 191(Suppl 1):S168-78

50 Hançali A, Ndowa F, Bellaji B, et al. Antimicrobial resistance monitoring in Neisseria gonorrhoeae and strategic use of funds from the Global Fund to set up a systematic Moroccan gonococcal antimicrobial surveillance programme. Sex Transm Infect 2013;89(Suppl 4):iv24-7

51 Rietmeijer KC, ed. The aetiology of STI syndromes in Zimbabwe. 17th IUSTI World Congress; May 2016; Marrakech. Marrakech, 2016.

52 Ministère de la Santé du Maroc. Estimations Nationales VIH /sida 2016: Rapport National 2016 sur la Mise en oeuvre de la declaration politique sur le VIH/SID. Rabat, 2016.

53 Johnston LG, Alami K, El Rhilani MH, et al. HIV, syphilis and sexual risk behaviours among men who have sex with men in Agadir and Marrakesh, Morocco. Sex Transm Infect 2013;89(Suppl 3):iii45-8.

54 Johnston LG. Integrated behavioral and biological surveillance survey among men who have sex with men (MSM) in Agadir and Marrakech, 2010-2011. Rabat: Kingdom of Morocco Ministry of Health and National STI/AIDS Programme, Joint United Nations Programme on HIVIAIDS, Global Fund, 2011.

55 Kuznik A, Habib AG, Manabe YC, et al. Estimating the public health burden associated with adverse pregnancy outcomes resulting from syphilis infection across 43 countries in sub-Saharan Africa. Sex Transm Dis 2015:42:369-75.

56 Woods CR. Gonococcal infections in neonates and young children. Semin Pediatr Infect Dis 2005;16:258-70.

57 Mahy M, Nzima M, Ogungbemi MK, et al. Redefining the HIV epidemic in Nigeria: from national to state level. AIDS 2014;28(Suppl 4):S461-7. 